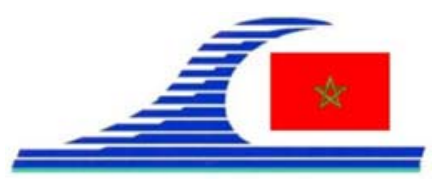

Conférence Méditerranéenne Côtière et Maritime

EDITION 2, TANGER, MAROC (2011)

Coastal and Maritime Mediterranean Conference

Disponible en ligne - http://www.paralia.fr - Available online

\title{
Analyse de la performance du modèle de lessivage RQSM
}

\author{
Éric CROBEDDU ${ }^{1}$, Saad BENNIS ${ }^{1}$
}

1. École de technologie supérieure, Département de génie de la construction 1100,

Notre-Dame Ouest, Montréal (Québec) H3C 1K3, Canada.

Saad.Bennis@etsmtl.ca

\section{Résumé :}

Le présent travail porte sur l'analyse de la performance et de la sensibilité du modèle conceptuel RQSM (Runoff Quality Simulation Model). Le modèle RQSM qui a pour objectif, la simulation du lessivage des matières en suspension considère que l'accumulation est infinie sur les surfaces perméables et imperméables. Il utilise l'énergie cinétique des précipitations pour décrire la mise en suspension des particules solides. Il modélise le transport des matières en suspension à l'aide de la théorie des systèmes linéaires.

Les charges de matière en suspension mesurées durant 22 évènements pluvieux enregistrés à l'exutoire du sous bassin (1) de l'arrondissement de Verdun au Canada et à l'exutoire de trois sous bassins de la ville de Champaign aux États Unis ont servi à valider le modèle RQSM.

\section{Mots-clés :}

Bassin urbain - Matière en suspension - Accumulation - Lessivage - Etude comparative

\section{Introduction}

Cet article présente un nouveau modèle conceptuel de lessivage des MES en milieu urbain. Le modèle RQSM se base sur une approche conceptuelle des phénomènes d'accumulation et de transport des particules solides par lessivage. Le modèle RQSM modélise d'une part le processus d'érosion par la mise en suspension des particules solides sous l'effet des précipitations et d'autre part le transport des particules solides par les eaux de ruissellement. De plus, la mise en suspension des particules solides sur les surfaces perméables et imperméables est traitée séparément. En se qui concerne la modélisation du lessivage des particules solides, elle repose sur l'hypothèse d'une réponse linéaire du bassin à une intensité de mise en suspension (BENNIS et al., 2001). Le modèle RQSM présente une formulation concise qui repose sur deux paramètres physiques de la qualité.

Le modèle proposé sera validé à l'aide de mesures de MES effectuées sur le terrain. Les résultats du modèle proposé seront comparés aux résultats du modèle exponentiel et du modèle de rating curve (TEMIMI \& BENNIS, 2002). 
La connaissance de la Mer :

un vecteur du développement durable en Méditerranée

\section{Représentations du processus d'accumulation}

La masse de solides accumulés est fonction du temps écoulé depuis le dernier événement pluvieux. Cette accumulation est généralement décrite à l'aide de l'équation suivante (HUBER \& DICKINSON, 1988) :

$\frac{d M a(t)}{d t}=A C C U-D I S P M a(t)$

où $M a$ représente la masse de particules solides accumulées $(\mathrm{kg})$; $A C C U$, le taux d'accumulation journalier $\left(\mathrm{kg} \mathrm{j}^{-1}\right)$; DISP, le coefficient de disparition des particules $(j-1)$.

\section{Formulation du modèle RQSM}

CROBEDDU et BENNIS (2011) ont démontré analytiquement que la relation la plus appropriée pour calculer l'énergie cinétique des précipitations en fonction de leur intensité est la suivante :

$K E(t)=\alpha I(t)^{\beta}$

où $K E$ représente l'énergie cinétique de la pluie $\left(\mathrm{J} \cdot \mathrm{m}^{2} \cdot \mathrm{h}^{-1}\right) ; \alpha$ et $\beta$, des paramètres liés aux processus microscopiques de formation des gouttes de pluie; $I$, l'intensité de la pluie $\left(\mathrm{mm} \mathrm{h}^{-1}\right)$.

L'hypothèse d'accumulation infinie conduit à exprimer l'intensité surfacique de mise en suspension selon la formule suivante (CROBEDDU et al., 2008) :

$m p_{\text {imp }}(t)=\frac{K p_{\text {imp }}}{3600}\left(\alpha I(t)^{\beta}\right)$

où $m p_{\text {imp }}$ représente l'intensité surfacique de mise en suspension des particules solides pour les surfaces imperméables $\left(\mathrm{kg} \cdot \mathrm{m}^{-2} \cdot \mathrm{s}^{-1}\right) ; K p_{i m p}$, le coefficient de mise en suspension des particules solides pour les surfaces imperméables $\left(\mathrm{kg} \mathrm{J}^{-1}\right)$.

L’intensité de mise en suspension surfacique pour les surfaces perméables est donnée par :

$m p_{\text {per }}(t)=\frac{K p_{\text {per }}}{3600}\left[\alpha(I(t)-f(t))^{\beta}\right]$

où $m p_{p e r}$ représente l'intensité surfacique de mise en suspension des particules solides pour les surfaces perméables $\left(\mathrm{kg} \cdot \mathrm{m}^{-2} \cdot \mathrm{s}^{-1}\right) ; K p_{p e r}$, le coefficient de mise en suspension des particules solides pour les surfaces perméables $\left(\mathrm{kg} \mathrm{J}^{-1}\right)$; $f$, la capacité d'infiltration des surfaces perméables $\left(\mathrm{mm} \mathrm{h}^{-1}\right)$. Le produit de convolution entre l'intensité de mise en suspension et la réponse du bassin est donné par l'équation suivante :

$$
m l(n)=\overbrace{\sum_{i=1}^{n \leq n}\left[m p_{i m p}(i) u_{i m p}(n-i+1)\right]}^{m l_{\text {imp }}(n)}+\overbrace{\sum_{i=1}\left[m p_{\text {per }}(i) u_{\text {per }}(n-i+1)\right]}^{m l_{\text {per }}(n)}
$$

avec : 


$$
u_{\text {imp }}(n-i+1)=\frac{10000 \mathrm{IMP} A}{t_{c}} \quad \text {, pour : } \quad 1 \leq(n-i+1) \leq t_{c}
$$

et :

$$
u_{p e r}(n-i+1)=\frac{10000(1-I M P) A}{t_{c}} \quad \text {, pour : } \quad 1 \leq(n-i+1) \leq t_{c}
$$

où $u_{\text {imp }}$ représente la réponse impulsionnelle des surfaces imperméables $\left(\mathrm{m}^{2} \mathrm{~min}^{-1}\right) ; u_{p e r}$, la réponse impulsionnelle des surfaces perméables $\left(\mathrm{m}^{2} \mathrm{~min}^{-1}\right)$; $\mathrm{A}$, la superficie du bassin versant (ha) ; IMP, la fraction de surface imperméable directement drainée ; $t_{c}$, le temps de concentration du bassin versant (min); $n$ et $i$, des indices de temps; $n_{p}$, l’indice final du vecteur des intensités de mise en suspension.

\section{Validation du modèle RQSM}

Les pollutogrammes de MES fournis par le modèle RQSM, le modèle exponentiel et le modèle de rating curve ont été comparés à 11 pollutogrammes mesurés (BENNIS, 2000). Les figures 1 et 2 montrent les résultats obtenus pour deux des bassins versants étudiés. Les trois modèles ont fourni un pollutogramme proche du pollutogramme mesuré pour 50 \% des évènements mesurés. L'erreur sur la masse de MES lessivée est inférieure à 30 \% pour 6 évènements simulés à l'aide du modèle RQSM et rating curve comparativement à 3 évènements simulés à l'aide du modèle exponentiel. Par conséquent, le modèle RQSM a modélisé la masse de MES lessivée de manière équivalente au modèle de rating curve mais mieux que le modèle exponentiel.

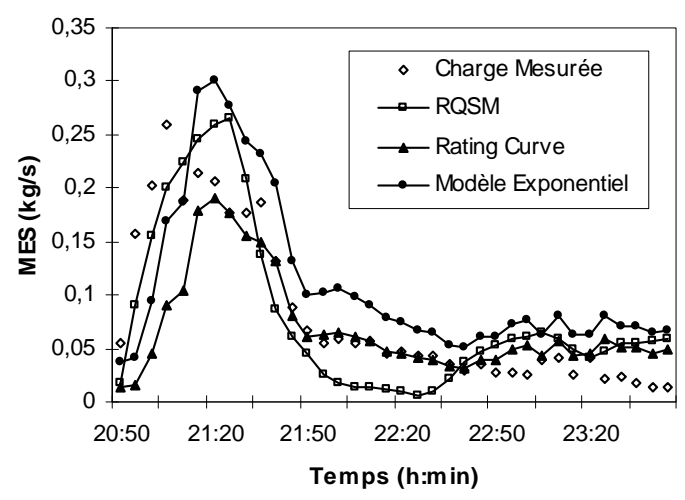

Figure 1. Pluie du 13 octobre 2000 sur le sous bassin (1) de Verdun.

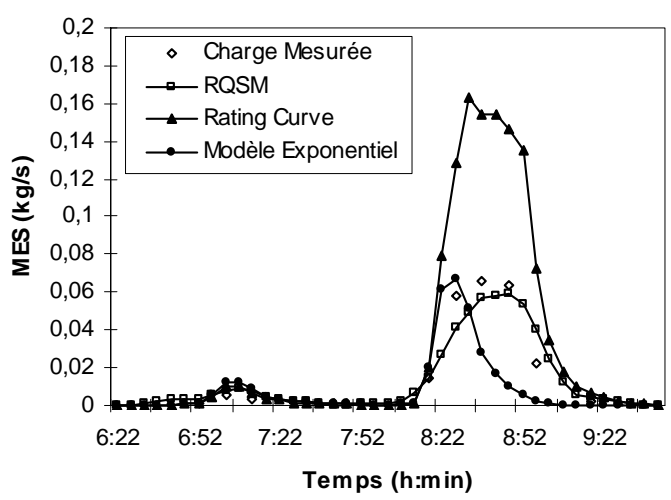

Figure 2. Pluie du 23 juin 1980 à South Mattis.

\section{Conclusion}

Le modèle RQSM a simulé les charges de MES mesurées, à l'exutoire de 4 sous bassins urbains situés en Amérique du Nord, avec un niveau de précision équivalent à celui de deux modèles conceptuels éprouvés. L'analyse de sensibilité du modèle RQSM a montré que deux paramètres avaient un impact significatif sur le pollutogramme simulé. 
La connaissance de la Mer :

un vecteur du développement durable en Méditerranée

Les résultats encourageants obtenus à l'aide du modèle RQSM permettent d'envisager une extension de son utilisation à d'autres polluants.

\section{Références bibliographiques}

BENNIS S. (2000). Caractérisation des eaux pluviales du secteur 1 de la ville de Verdun. Rapport Technique : École de technologie supérieure, Ville de Verdun et BGH Planning.

BENNIS S., HAJJI T., BERNARD F. (2001). Phénomène du premier flot: mythe ou réalité ? Vecteur Environnement, section scientifique, 34(1), pp 34-42.

CROBEDDU E., BENNIS S. (2011). A new model for simulating TSS washoff in urban areas. Journal of Applied and Environmental Science. Volume 2011, ID318765, pp 1-9. doi:10.1155/2011/318765

CROBEDDU E., ALLALI S., BENNIS S. (2008). Washoff model of total suspended particles in urban areas. Conference: 9th International Conference on Modelling, Monitoring and Management of Water Pollution: Univ Alicante, Alicante, Spain.

HUBER W.C., DICKINSON R.E. (1988). Storm Water Management Model, Version 4, User's Manual. U.S. EPA, Athens, Georgia, EPA/600/3-88/001.

TEMIMI S., BENNIS S. (2002). Prévision en temps réel des charges de polluants dans un réseau d'assainissement urbain. Revue des Sciences de l'Eau, 15(3), pp 661-675. 